\title{
A light-weight available bandwidth inference methodology in a queueing analysis approach
}

\author{
Xiaojun Hei ${ }^{\dagger}$, Brahim Bensaou ${ }^{\ddagger}$ and Danny H.K. Tsang ${ }^{\dagger}$ \\ ${ }^{\dagger}$ Dept. of Electrical \& Electronic Engineering \\ ${ }_{\ddagger}^{\ddagger}$ Dept. of Computer Science \\ Hong Kong University of Science and Technology \\ Clear Water Bay, Kowloon, Hong Kong
}

\begin{abstract}
End-to-end available bandwidth estimation is important in understanding network congestion and enhancing service quality. In this paper, we investigate a light-weight probing method for available bandwidth measurement in a queueing analysis approach. Unlike the self-congestion based measurement approach, a light-weight probing technique infers the available bandwidth along a path without congesting the routers along the path. Of particular interest in our investigations, is the Squared Coefficient of Variation (SCV) of the inter-departure process of a periodic probing stream. We analyze approximately the departure process of this probing stream. Simulation results indicate that the proposed hybrid approximation can provide good estimates of the SCV of the probing stream regardless of the stochastic behavior of the arrival process of the cross traffic. Given a measured SCV, inverting this approximation infers the load of the cross traffic on the congested link.
\end{abstract}

\section{INTRODUCTION}

Available bandwidth estimation has become an active research topic in the past several years due to a wide area of application. Various end-to-end active probing techniques have been proposed in the past several years. They can be classified into two categories, self-congestion techniques [1][3], and model-based techniques [4]-[6]. Despite the good characteristics, claimed by proponents of the self-congestion approach, such as accuracy, simplicity, speed, robustness, and so on, the congestion introduced by the probing stream inevitably changes the load along the path in such a way that TCP connections along the path sometimes suffer dramatically. Conversely, in the model-based approach, the tool is operated when the path is not congested. This brings forth the possibility of a light-weight available bandwidth estimation technique.

In the model-based available bandwidth measurement, the performance metrics of the active probing stream fall into two categories, loss-related metrics [4], [5] and delay-related metrics [6], [7]. The buffer size along the path has a significant impact on loss-based measurements. When the buffer is small, the loss measurements appear to fluctuate significantly; when the buffer is large, the loss is hardly observable while guaranteeing non-intrusiveness. Differently, delay-based measurement, (in particular, based on the delay variation information), provides a more advantageous approach in the available bandwidth estimation [7].

Our contribution in this paper is two fold: 1) We propose and evaluate a light-weight probing method for the available bandwidth estimation based on the queueing analysis of the delay variation of a periodic probing stream when the endto-end path is not congested $(\rho<1)$. To our knowledge, model-based available bandwidth measurement using delayvariation has not been investigated, except our previous work for Poisson probing in [6], [7]. 2) We propose a light traffic approximation to analyze the SCV of the departure process of the periodic stream, namely, hybrid approximation, which is also verified via simulations.

The rest of the paper is organized as follows. First, in Section II some related works are outlined. Next, in Section III we present a general end-to-end measurement framework of which, the available bandwidth estimation problem can be considered as one application. In Section V, our evaluation of the proposed model is presented using extensive simulations. Finally, conclusions are drawn in Section VI.

\section{RELATED WORKS}

Pathload [1] and Initial Increasing Gap (IGI) [2] are two available bandwidth measurement methodologies that use a self-congestion approach. By trying different probing rates using a binary search, a reasonable estimate of the available bandwidth can be found in Pathload. In [2] $\mathrm{Hu}$ and Steenkiste reported that a turning point exists at which the average input gap equals the average output gap, and the average rate of the packet train equals the available bandwidth on the bottleneck link. In [3], TOPP shared the same nature as a self-congestion probing method. The difference is that TOPP utilized the throughput measurement to identify a throughput transition point when the path load $\rho$ is 1. In [8], Strauss et al. improved the probe gap model of [2] by setting the inter-gap time between two probe pairs to be exponentially distributed. Their measurement tool, Spruce, shows better accuracy than IGI; however, Spruce essentially retains the self-congestion approach.

The model-based measurement method most closely related to our work is [4]. Two inference (queueing) models, $M+$ $M / M / 1 / K$ and $M+M / D / 1 / K$, were developed for a single congested link, to simultaneously estimate the background traffic intensity and the buffer size of the congested link. Following a similar approach of [4], cross traffic characteristics were inferred based on the loss process analysis of the $M M P P / M / 1 / N$ queue in [5]. Experiments [6], [7] have 
shown that measurements using packet loss are very sensitive to the size of the buffer along the end-to-end path. Such buffer size is practically unknown.

\section{A General End-To-End MeAsurement FRAMEWORK}

The essence of active measurement is to identify an "effect" in order to establish the relationship between the target measurement metric, i.e., the available bandwidth, and the measurable performance metrics of the active probing stream at the receiver. With a well established relationship, the available bandwidth can be inferred from the performance measurement of the active probing stream. For end-to-end available bandwidth measurement, we present a general measurement framework, which is depicted in Fig. 1. This framework consists of two sub-problems, the problem modelling of an end-to-end path and the problem of inferring the unknown parameters of this path model. In the modelling problem, the end-to-end path is modelled as a series of tandem queues with a superposition of two arrival processes, the probing stream and the cross traffic stream. This network model is determined by a set of determinable or controllable parameters, $\Theta$, of the probing actions and a set of unknown parameters, $X$, which include the target measurement metrics, i.e., the available bandwidth.

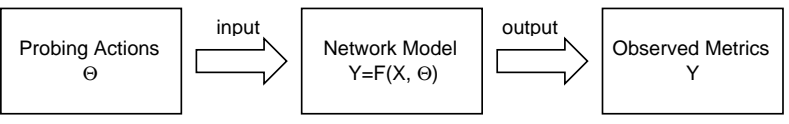

Fig. 1. A general end-to-end model-based measurement framework

The output of the measurement process is the observed metrics $Y$. With an appropriate model, the relationship between $Y$ and $(X, \Theta)$ can be established, i.e., $Y=F(X, \Theta)$. Therefore, the performance of an active probing stream from the sender to the receiver can be predicted given all the parameters of this queueing model. As an inverse process, in the inference problem, the parameters of this queueing model, that are of particular interests, including path capacity and available bandwidth, can be estimated by measuring the performance of this probing stream. In a mathematical form, $X=F^{-1}(Y, \Theta)$. Relatively accurate tools for capacity measurement have been proposed in [9]. Our focus is on the estimation of the cross traffic intensity under the assumption that the path capacity is known, which is provided for instance by the packet-pair technique.

Probing sequences with two types of inter-arrival time distribution are commonly used: Poisson probe sequences in RFC2330, periodic probe sequences in RFC3432. In this study, we focus on periodic probing. Due to the impact of the cross traffic, a periodic probing stream with a constant packet size is no longer periodic when it arrives at the receiver. This alteration effect on the inter-departure time between consecutive probing packets can be characterized using Squared Coefficient of Variation (SCV). Thus, in a real measurement system, given the measured SCV of the probing stream, inverting the function $F$ helps in inferring the load of the cross traffic on an end-to-end path.

\section{ANALYSIS OF THE DELAY PROCESS IN $D+M / G I_{i} / 1 / \infty$}

With the assumptions that the cross traffic is a simple Poisson arrival process and that there exists only a single bottleneck along the end-to-end path, the active periodic probing problem can be formulated into a queueing analysis problem with respect to the performance metrics of the probing stream. The system is modelled as a single server, finite capacity queue operating in continuous time, which accepts two types of arrivals, probing packet arrivals and cross traffic packet arrivals (Fig. 2). The probing packet arrival process is deterministic. Its arrival rate is denoted as $\lambda_{1}$ and the service rate is $\mu_{1}$. The arrival rate of the cross-traffic process is denoted as $\lambda_{2}$ and the service rate as $\mu_{2}$. The traffic intensity $\rho=\rho_{1}+\rho_{C T}=$ $\frac{\lambda_{1}}{\mu_{1}}+\frac{\lambda_{2}}{\mu_{2}}$. Denote the buffer size $K$ including the one in service $(K \geq 1$ packets). The corresponding queueing model can be represented as $D+M / G I_{i} / 1 / K$.

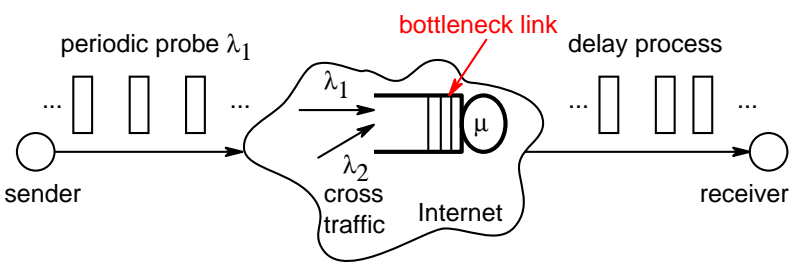

Fig. 2. The available bandwidth inference model.

The analysis of the delay process of the probing stream in the $D+M / G I_{i} / 1 / K$ queue is difficult because packet dropping may destroy the delay pattern. Under the assumption that the buffer size is infinite, the original queue becomes $D+M / G I_{i} / 1 / \infty$. As shown in [7], the impact of the packet dropping on the delay pattern of the probing stream is minimal because our probing load is very small by setting very small probing packet size ( $L=40$ bytes).

Fig. 3 shows the time diagram of the delay process in $D+$ $M / G I_{i} / 1 / \infty$. Denote by $C_{n}$ the $n$-th probing packet arrival and by $X_{n}$ its service time. Let $I_{n}$ be the inter-arrival time between the $n$-th and $(n+1)$-st probing packets, and let $D_{n}$ be the corresponding inter-departure time. The number of arrivals from the cross traffic stream which occur during $I_{n}$, is $N_{y}$. $\left\{Y_{j}\right\}$ is the service time for the $j$-th packet from the cross traffic stream that arrives after $C_{n}$ and before $C_{n+1}$.

The delay gap ratio is defined as the ratio between the average inter-departure time $D_{n}$ and the average inter-arrival time $I_{n}$ of the probing packets. This ratio provides first-order delay information of the delay process. Following a similar queueing analysis approach in [7], we derived delay gap ratio in (1).

$$
\text { GapRatio }=\frac{E\left[D_{n}\right]}{E\left[I_{n}\right]}=\left\{\begin{array}{l}
1, \rho<1 \\
\rho_{1}+\rho_{C T}, \rho \geq 1
\end{array}\right.
$$

From (1), there exists a transition point, when the aggregate load $\rho=1$. When $\rho<1$, the average inter-departure 


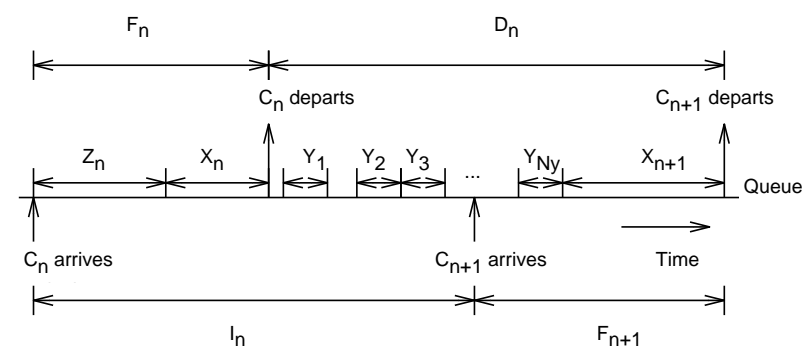

Fig. 3. The time diagram of the delay process in $D+M / G I_{i} / 1 / \infty$.

time $\left(E\left[D_{n}\right]\right)$ equals the average inter-arrival time $\left(E\left[I_{n}\right]\right)$ of the probing packets. In [2], the IGI algorithm is essentially utilizing this property of the delay process of the probing stream to identify this transition point, when the probing load is equal to the available bandwidth. Note that the identification of this transition point requires the probing rate be large enough to congest the path $(\rho \geq 1)$ for some period of time. The existence of this gap transition point also explains the basic principle used in Pathload [1]. When the delay gap is larger than 1, it equivalently indicates that the one way delay of the probing packets exhibits a strong increasing trend. When the delay gap equals 1 , this one way delay increasing trend is practically not observable.

The delay variation is defined as $J_{n}=D_{n}-I_{n}$. Because $I_{n}$ is constant for a periodic stream, the task of characterizing $J_{n}$ can be reduced to finding the variation of $D_{n}$. When the path load is far less than 1 , the average output gap equals the average input gap regardless of the types of arrival processes of the cross traffic and the traffic intensity. Therefore, this first order delay information of the departure process cannot provide interesting information about the exact load of the cross traffic when $\rho<1$. Therefore, we turn to investigate the second order characteristic of the departure process of the probing stream.

The SCV is a dimensionless metric to characterize the delay variation process of the probing stream. It is defined as the ratio between the variance of the inter departure time and the squared average inter departure time of two probing packets, $S C V_{D_{n}}=\frac{\operatorname{Var}\left[D_{n}\right]}{E^{2}\left[D_{n}\right]}$. By the queueing analysis of [10], there are various influential factors upon $S C V$, including the variance of the probing stream, the variance of the packet size of the cross traffic and the load of the cross traffic. In the terminology of Section III first two are the determinable parameters $(\Theta)$ and the last one is the target measurement metric $(X)$.

When probing packets traverse the network along a path in the multi-class queue as in Fig. 2, the departure process of the probing stream is determined by its arrival process and the disturbance from the cross traffic. An exact calculation of $S C V_{D_{n}}$ is difficult and only complex numerical solutions exist, which is not suitable for our online measurement; hence, we turn to an approximate analysis of $S C V_{D_{n}}$.
From [11] for a two-class queueing model, we have

$$
C_{1}^{D}=\rho_{1}^{2} C_{1}^{S}+\rho_{2}^{2}\left(p_{1} / p_{2}\right)\left[C_{2}^{S}+C_{2}^{A}\right]+\left(1-\rho_{1} \rho+\rho_{1}^{2}\right) C_{1}^{A},
$$

where, $C_{i}^{D}$ is the SCV of the departure process for class $i, C_{i}^{S}$ is the SCV of the service process for class $i, C_{i}^{A}$ is the SCV of the arrival process for class $i, \rho_{i}$ is the traffic intensity for class $i$ and $p_{i}$ is the probability that an arrival is from class $i$.

Substitute the periodic probing stream in Fig. 2 with a Poisson probing stream and maintain the other parameters unchanged. The $D+M / G I_{i} / 1 / \infty$ queue turns into $M_{1}+$ $M_{2} / G I_{i} / 1 / \infty$, which was exactly analyzed in [12]. Our idea of analyzing the departure process of the periodic stream in $D+M / G I_{i} / 1 / \infty$ is to identify the relationship between these two queueing systems. We proposed an approximate analysis, namely, "hybrid approximation". $S C V_{D_{n}}^{\text {Periodic }}=$ $S C V_{I_{n}}^{\text {Periodic }}+S C V_{C T}=S C V_{D_{n}}^{\text {Poisson }}-\left(1-\rho_{1} \rho+\rho_{1}^{2}\right)$. Note that $S C V_{I_{n}}^{\text {Poisson }}=1$ and $S C V_{I_{n}}^{\text {Periodic }}=0$; therefore, $S C V_{D_{n}}^{\text {Periodic }}=S C V_{I_{n}}^{\text {Periodic }}+S C V_{C T}=S C V_{D_{n}}^{\text {Poisson }}-$ $\left(1-\rho_{1} \rho+\rho_{1}^{2}\right)$. A heavy traffic approximation approach [10], namely, Fischer-Stanford approximation, can also be employed to analyze the delay process of this periodic probing stream.

\section{Simulation Results}

The assumption of the Poisson-type cross traffic leads to a tractable mathematical treatment; however, it is also important to test the robustness of the proposed model when this assumption does not hold. We evaluated our probing strategy via simulation. The whole simulation model was constructed based on SimLib 2.2 [13]. With different types of arrival processes of the cross traffic, as shown in Fig. 4, the SCV increases when the normalized probing load increases. For different types of the arrival processes of the cross traffic, the probing stream has a similar SCV performance; therefore, the analysis of the per-class departure process for the Poisson-type cross traffic can be used to infer the non-Poisson type cross traffic without much loss in the accuracy.

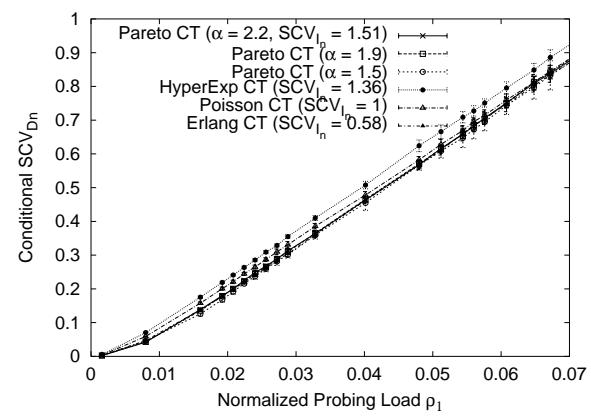

Fig. 4. Relationship between the conditional $S C V_{D_{n}}$ and the normalized probing load $\rho_{1}$ via simulations with different types of cross traffic arrival processes and the cross traffic load $\rho_{C T}=0.62$.

In Fig. 5(a), the proposed hybrid approximation is shown to match well the simulation results. In Fig. 5(b), the accuracy of the Fischer-Stanford approximation is evaluated when the probing packet size is $L=40$ bytes. The proposed hybrid approximation outperforms the Fischer-Stanford approximation. 
From Fig. 5, $S C V_{D n}$ is largely influenced by the load of the cross traffic, when $\rho<1$. This effect can be used to infer the available bandwidth without congesting the path. The exact inference can be accomplished numerically using a standard non-linear equation solver, since $F^{-1}$ is already known [6]. After this inference procedure, an upper and a lower bound of the available bandwidth estimation can be obtained.

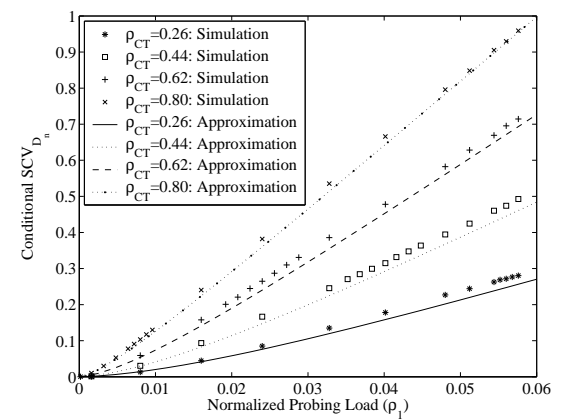

(a) Hybrid approximation

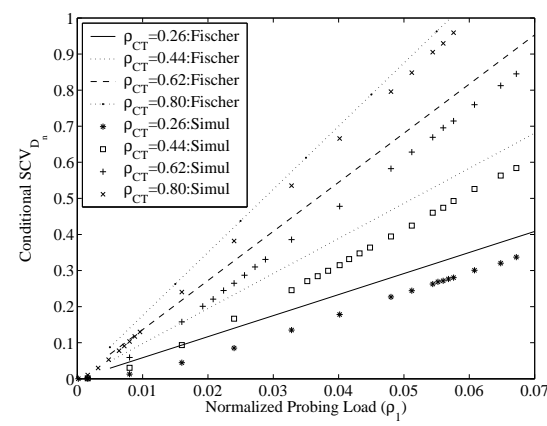

(b) Fischer-Stanford approximation

Fig. 5. The relationship between $S C V_{D_{n}}$ and the normalized probing load $\rho_{1}$ with Poisson-Type cross traffic and probing packet sizes $L=40$ bytes using the hybrid approximation and the Fischer-Stanford approximation.

We also investigated the impact of the multi-hop effects upon the accuracy of the SCVs of the probing stream. The topology used in simulation is a 5-hop path, depicted in Fig. 6. The probing packets enter the path at link 0 and exit at link 4. The tight link has capacity $C_{t}$ and available bandwidth $A_{t}$. The other links are referred as non-tight links, and they all have the same capacity $C_{n t}$ and available bandwidth $A_{n t}$. Crosstraffic is generated at each link from ten random sources. The arrival process of the cross traffic follows a Pareto distribution with the shape variable $\alpha=1.5$. The cross-traffic packet sizes are obtained from a Poisson distribution with a mean value of 441 bytes. By controlling the arrival rate of the cross traffic, the link load can be configured. The probing packet size is 40 bytes.

We consider a single congested link situation. Link 2 is the tight link $\left(C_{t}=10 \mathrm{Mbps}\right)$, and the other four links are nontight links $\left(C_{n t}=50 \mathrm{Mbps}\right)$. Four cross traffic load scenarios at link 2 were stimulated. The cross traffic load at other links are configured the same as the one at link 2 . Therefore, only link 2 is mostly congested and the other four links are lightly loaded. In Fig. 7, the conditional $S C V_{D_{n}}$ of the probing stream at the outport of each link is plotted. At link 0,1 , the overall load is light; therefore, the $S C V_{D_{n}}$ of the probing stream is only slightly higher than 0 . The $S C V_{D_{n}}$ at the outport of link 0 and link 1 are almost the same as that of the periodic probing stream $\left(S C V_{I_{n}}=0\right)$. This can be explained by the light approximation in [14]. After the bottleneck at link 2, we observe a significantly increased $S C V_{D_{n}}$ and this $S C V_{D_{n}}$ is preserved along link 3 and 4 and is finally measured at the receiver with little distortion.

In Fig. 8, the accuracy of the intensity inference of the cross traffic at the receiver using the hybrid approximation for $D+M / G I_{i} / 1$ is depicted for Pareto cross traffic $(\alpha=1.5)$. The estimation accuracy improves with the normalized probing load increasing. However, further increasing the probing load does not improve the inference accuracy significantly. The reasons can be two fold. One is that the Pareto-type cross traffic already breaks the assumption of Poisson cross traffic in $D+M / G I_{i} / 1$. The other may be due to the nature of the approximate calculation for $S C V_{D_{n}}$ of the periodic probing stream. Nevertheless, when the normalized probing load is larger than 0.02 , the relative estimation error is less than $10 \%$.

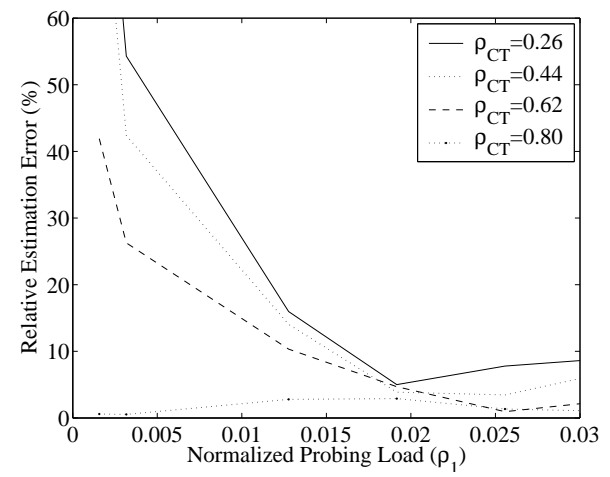

Fig. 8. Accuracy of the periodic probing using the hybrid approximation for $D+M / G I_{i} / 1$ with Pareto-type cross traffic $(\alpha=1.5)$ and the probing size $L=40$ bytes.

\section{CONCLUSION}

In this paper we proposed a light-weight model-based probing methodology for available bandwidth measurement based on the analysis of the departure process of an active periodic probing stream. Unlike self-congestion based available bandwidth measurement, our measurement can infer the available bandwidth along a path when the load of the path is less than 1 .

Both the first-order and second-order delay properties of the probing stream are of interest. The gap ratio between the average inter departure time and the average inter arrival time of two probing packets can serve as the criteria for determining the load region of an end-to-end path. By reducing the probing rate, the measurement can be possibly operated when the 


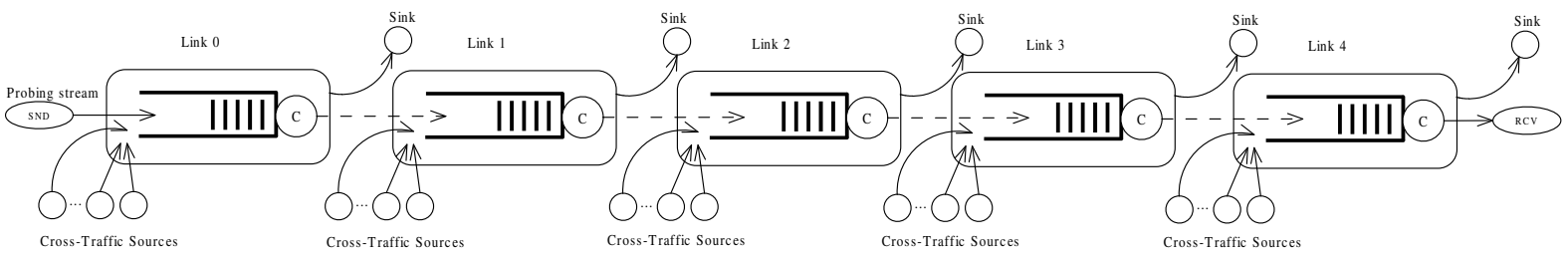

Fig. 6. A multi-hop topology.

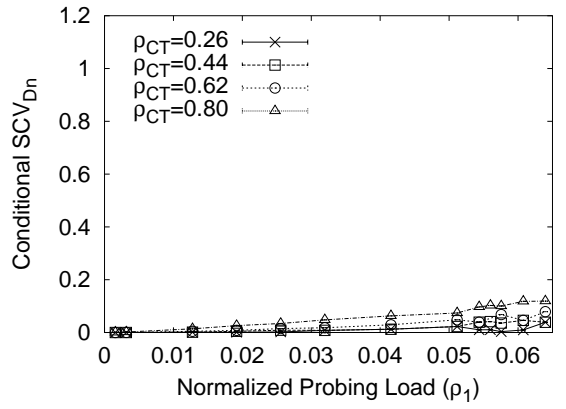

(a) Link 0

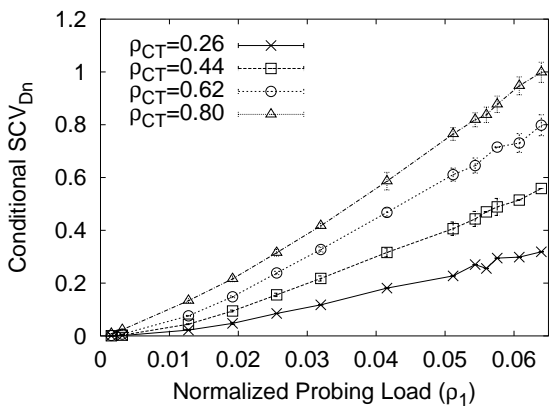

(c) Link 2

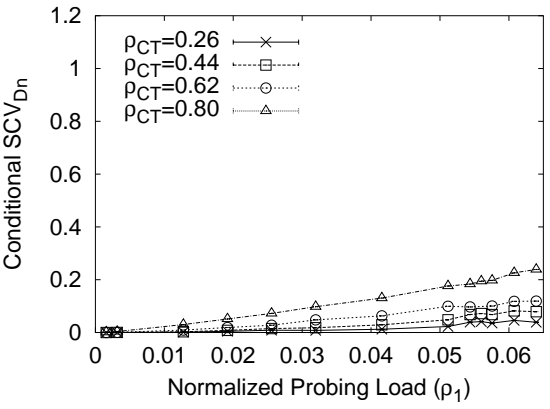

(b) Link 1

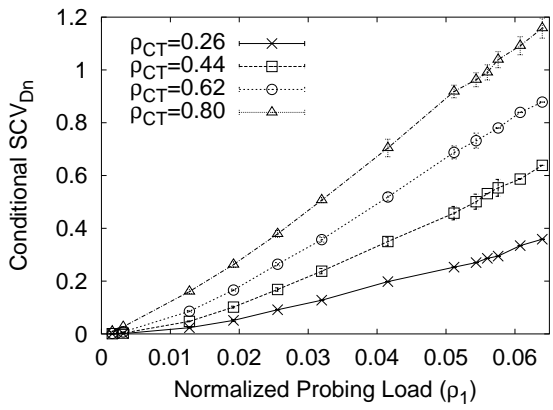

(d) Link 4

Fig. 7. The relationship between the conditional $S C V_{D_{n}}$ and the probing rate $\lambda_{1}$ with Pareto-type $(\alpha=1.5)$ cross traffic and the buffer size=500KBytes.

load of the path is less than 1 and hence the model-based available bandwidth inference can be conducted based on the second-order characteristic of the departure process of the probing stream. The SCV based model has a good accuracy of available bandwidth estimation for a single bottleneck. For multiple congested links, the exact queue analysis is difficult; therefore, we are more interested in investigating the approximate analysis of the departure process of these tandem queues in a decomposition approach.

\section{REFERENCES}

[1] M. Jain and C. Dovrolis, "End-to-end available bandwidth: measurement methodology, dynamics, and relation with TCP throughput," IEEE/ACM Transactions on Networking, vol. 11, no. 4, pp. 537-549, 2003.

[2] N. Hu and P. Steenkiste, "Evaluation and characterization of available bandwidth probing techniques," IEEE JSAC, vol. 21, no. 6, 2003.

[3] B. Melander, M. Bjorkman, and P. Gunningberg, "A new end-to-end probing and analysis method for estimating bandwidth bottlenecks," in Proceedings of GLOBECOM, 2000.

[4] S. Alouf, P. Nain, and D. Towsley, "Inferring network characteristics via moment-based estimators," in Proceedings of INFOCOM, 2001.
[5] K. Salamatian, B. Bruno, and T. Bugnazet, "Interpretation of losses observed on the Internet by inferring traffic characteristics," in Proc. of the DIMACS Workshop on Internet and WWW Measurement, Mapping and Modeling, 2002.

[6] X. Hei, D. Tsang, and B. Bensaou, "Available bandwidth measurement using Poisson probing on the Internet," in Proc. IPCCC, 2004.

[7] _ " "On model-based available bandwidth measurement on the Internet," in Proc. SPECTS'04, pp. 423-430, 2004.

[8] J. Strauss, D. Katabi, and F. Kaashoek, "A measurement study of available bandwidth estimation tools," in Proc. IMC, pp. 39-44, 2003.

[9] C. Dovrolis, P. Ramanathan, and D. Moore, "What do packet dispersion techniques measure?" in Proceedings of INFOCOM, pp. 905-14, 2001.

[10] W. Fischer and D. Stanford, "Approximations for the per-class waiting time and interdeparture time in the $\sum_{i} G I_{i} / G_{i} / 1$ queue," Performance Evaluation, vol. 14, no. 2, pp. 65-78, 1992.

[11] W. Whitt, "Towards better multi-class parametric-decomposition approximations for open queueing networks," Annals of Operations Research, vol. 48, no. 1-4, pp. 221-48, Jan. 1994.

[12] D. Stanford and W. Fischer, "The interdeparture-time distribution for each class in the $\sum_{i} M_{i} / G_{i} / 1$ queue," Queueing Systems, vol. 4, no. 3, pp. 179-91, July 1989 .

[13] IKR. University of Stuttgart, IKR Simlib library 2.2.

[14] W. Whitt, "A light-traffic approximation for single-class departure processes from multi-class queues," Management Science, vol. 34, no. 11 , pp. 1333-1346, 1988. 